\title{
The influence of terahertz radiation on biochemical metabolism of blood in the experiment
}

\author{
A.G. Soloveva, P.V. Peretyagin, A.G. Polyakova, N.V. Didenko \\ Federal State Budgetary Educational Institution of Higher Education «Privolzhsky Research Medical University» of the Min- \\ istry of Health of the Russian Federation, Nizhny Novgorod, Russia, sannag5@ mail.ru
}

In recent years, close attention has been paid to studies related to the impact of electromagnetic radiation (EMR) terahertz $(\mathrm{THz})$ range on the living organism. Until now, the final idea of the ways and physical and chemical mechanisms of EMR THz action has not been formed, so it is important to identify all possible "points of application" of such an impact on biological systems [1]. Because the level of lipid peroxides reflecting the integrity of biological membranes changes under the action of different EMR ranges [2], and the antioxidant system controls the intensity of free radical processes, providing protection of cells and tissues [3], these systems were chosen to detect changes caused by $\mathrm{THz}$ radiation.

The aim of the work was to study the effect of electromagnetic radiation of the terahertz range on the functional and metabolic parameters of rat blood in the process of reparative regeneration.

\section{Materials and methods}

The experiment was carried out on 15 male Wistar rats weighing 250-300 g. in accordance with the requirements of the Geneva Convention "International Guiding Principles for Biomedical Research Involving Animals" (Geneva, 1990). The impact of EMR was carried out in the direct sound irradiation EMR $\mathrm{THz}(110-170 \mathrm{GHz})$ from the experimental emitter apparatus "AMFIT" (N.Novgorod).

Animals were divided into 3 equal groups: 1 - intact healthy rats, 2 - control - operated animals without any effects, 3 - operated animals with irradiation EMR THz at a dose of $0.12 \mathrm{MJ}$ within the next 7 days (10 minutes 1 time per day). The impact was carried out on the base area of the displaced flap, coinciding with the localization of the skin projection of the center of vegetative regulation. Surgical intervention in 2 and 3 groups of rats and withdrawal from the experiment on day 7 after the operation was carried out under intramuscular anesthesia (Zoletil + Xyla).

Blood stabilized with sodium citrate (1:9) was used for the studies. Glucose and lactate concentrations were measured on a Super GL ambulance device (Germany) in plasma and red blood cells. The activity of lactate dehydrogenase (LDH) in direct (LDHdir) and reverse (LDHrev) reactions was determined on the spectrophotometer Power Wave XS (Bio-Tek, USA). The total content of nitric oxide metabolites was determined by the method of L.C.Green with coauthors in the modification of V. A. Metelskaya and N. G. Gumanova [4].

The intensity of lipid peroxidation (LPO) of a biological object by the level of malonic dialdehyde (MDA) in plasma and hemolysate of washed erythrocytes (1:10) [5], the activity of superoxide dismutase (SOD) [6] in hemolysate of washed erythrocytes
(1:10) and catalase [7] in erythrocytes were determined by spectrophotometry.

The state of microcirculation and the degree of microcirculatory insufficiency with the analysis of the parameter of microcirculation (PM) characterizing the degree of tissue perfusion by blood, using wavelet analysis, were evaluated according to laser Doppler fluorometry on the analyzer of capillary blood flow LAKK-M (Russia).

Statistical processing was carried out using the program Statistica, version 6.0, using the Student criteria. Differences were considered statistically significant at $\mathrm{p}<0.05$.

\section{Results}

In animals of group 2 the activation of LPO in erythrocytes was detected, MDA concentration increased by $20 \%$ compared to intact animals (Fig.1). In the erythrocytes of control animals compared with the intact group a compensatory increase of catalase specific activity on $17 \%(\mathrm{p}=0.02)$ was registered, but a SOD activity decreased on $16 \%(\mathrm{p}=0.003$ ) (Fig.2). It indicated about an insufficient degree of compensation of the LPO by the antioxidant system in the operated animals.

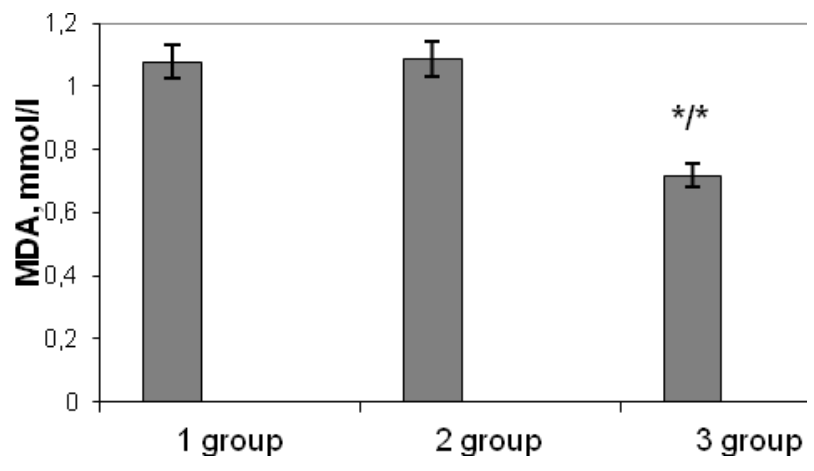

Fig.1. The concentration of malonic dialdehyde in the blood plasma of healthy and operated animals under the influence of EMR THz.

Note: * - differences are statistically significant compared to group $1 ; * *$ - differences are statistically significant compared to group 2.

The decrease of the intensity of LPO in plasma under the influence of THz-irradiation was observed. MDA concentrations in plasma decreased by $34 \%$ (relative to both comparison groups) under the influence of EMR THz.

The activity of enzymes of bioradical protection increased in the experimental group after irradiation of EMR THz. The most positive dynamics was observed for the SOD. SOD activity after surgery in the experimental group of animals increased by $30 \%$ 
$(p=0.018)$ compared to the control and reached the indicator of healthy animals. The activity of catalase also increased by $12 \%(\mathrm{p}=0,025)$ compared to the rate of intact rats under the influence of irradiation. It reduces the free radical forms of oxygen and inactivates LPO. Consequently, $\mathrm{THz}$ exposure in the noise mode of radiation has an antioxidant effect through the activation of SOD and catalase, which, in turn, inhibit the release of catecholamines from the nerve endings and adrenal glands, as well as the action of these monoamines at the postsynaptic level [8]. Therefore, it is possible that one of the mechanisms that provide a decrease in the intensity of LPO under the action of EMR THz is the suppression of hyperactivity of one of the most important stressimplementing systems of the sympathoadrenal system.

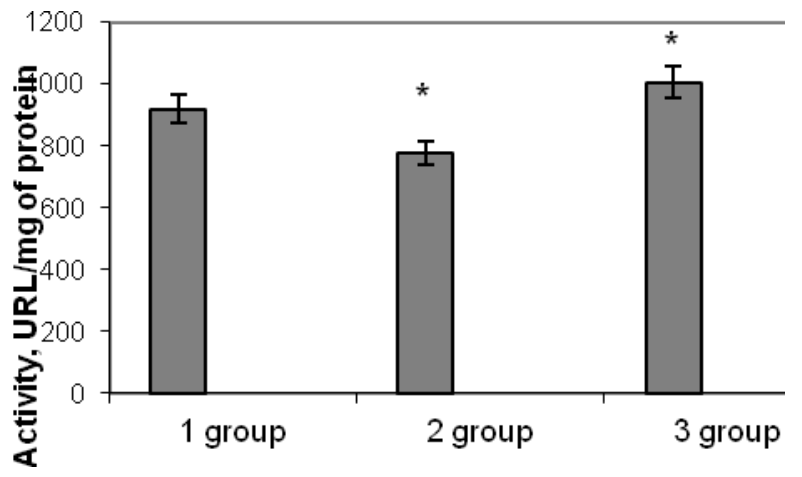

Fig.2. Activity of blood superoxide dismutase in healthy and operated animals under the influence of EMR THz

Note: $*$ - differences are statistically significant compared to group 1 ; $* *$ - differences are statistically significant compared to group 2 .

Thus, in the experimental group of animals the predominance of antioxidant activity over the processes of lipoperoxidation was observed. It may indicates the inhibition of the biological oxidation system due to the suppression of reactive oxygen species under the influence of EMR THz. In response to the irradiation of the projection of the center of vegetative regulation, apparently, there is a neurohumoral activation of the antioxidant system, which blocks the processes of LPO. The revealed change in the direction of LPO processes under irradiation may be due to changes in the structure (conformation) of the cell surface of membrane components due to the weakening of hydrophobic bonds [1].

There was an increase in glucose and lactate in rats of the control group in plasma and erythrocytes. An increase in glucose concentration can contribute to a significant formation of free radicals through glycation reactions and autooxidation of glucose. The decrease of LDHdir on $33 \%$ in trauma in comparison with healthy animals.

EMR irradiation of $\mathrm{THz}$ in the noise range 110$170 \mathrm{GHz}$ contributed to a decrease in the concentration of lactate in plasma and erythrocytes by $34 \%$ and $51 \%$, respectively, compared with the animals of the control group. It was shown that under the influence of EMR THz activity of LDHdir increased on $47 \%$, LDHrev - on $23 \%$ compared to the LDH activity of the rats without irradiation. In addition, it is possible that electromagnetic radiation promotes the use of lactate in carbohydrate metabolism in the formation of 2,3-diphosphoglycerate, the main source of energy in the body.

The zone of irreversible vascular changes in the flap at animals of 3 group after irradiation of EMR with a wide frequency range $(110-170 \mathrm{GHz})$ containing spectra of the main metabolite molecules, was less pronounced in comparison with the control.

Since the most common among all microcirculatory disorders are local vascular spasm and decrease in blood flow rate, the registered preliminary data on animals confirm the spasmolytic mechanism of action of electromagnetic radiation of the $\mathrm{THz}$ range on the smooth muscle vascular wall of animals. At the same time, an increase in the content of nitrites and nitrates in the blood serum was revealed after irradiation of EMR THz.

\section{Conclusion}

The results showed that EMR of $\mathrm{THz}$ with a noise range of $110-170 \mathrm{GHz}$ in ischemia led to an increase in the antioxidant status of the blood, as well as inhibition of peroxide states. It was shown that EMR THz of noise range 110-170 $\mathrm{GHz}$ increased the energy metabolism of blood in case the tissue ischemia.

\section{References}

1. Bajinyan, S.A., Meliksetyan, A.M., Malakyan, M.G. et al. // Millimeter waves in biology and medicine 2003. V.32, No 4. P. 12.

2. Gapeev, A.B., Cheremis, N.K. Mechanisms of biological action of electromagnetic radiation of extremely high frequencies at the cellular level // Biomedical technologies and Radioelectronics. 2007. No. 2-4. P. 44-61.

3. Ulashchik, V.S. Electromagnetic waves of the terahertz range and their therapeutic and prophylactic use // Questions of balneology, physiotherapy and physical therapy. 2007. No. 4. P. 3-7.

4. Metel'skaya, V.A., Gumanova, N.G. Screening method for determining the level of nitric oxide metabolites // Clinical laboratory diagnostics. 2005. No. 6. P. 15-18.

5. Uchiyama, M., Mihara, M. Analyt. Bbiochem. 1978. No. 86. P. 271.

6. Sirota, T.V. A new approach in the study of the process of adrenaline auto-oxidation and its use for measuring the activity of superoxide dismutase // Questions of medical chemistry. 1999. V. 45, No. 3. P. 109-116.

7. Sibgatullina, G.V., Haertdinova, L.R., Gumerova, E.A. et al. Methods for determining the redox status of cultivated plant cells: teaching aid. Kazan, Kazan (Volga) Federal University, 2011.

8. Chuyan, E.N., Ravaeva, M.Y., Nikolskaya, V. A. et al. Scientific notes of Taurida national University. V.I. Vernadsky. Series biology, chemistry. 2013. V. 65, No. 26. P. 223. 